\title{
Existence and iteration of positive solution for fractional integral boundary value problems with $p$-Laplacian operator
}

Ying $\mathrm{He}^{1 *}$ and $\mathrm{Bo} \mathrm{Bi}^{1}$

"Correspondence:

heying65332015@163.com 'School of Mathematics and Statistics, Northeast Petroleum University, Daqing, P.R. China

\section{每 Springer}

\begin{abstract}
This paper is concerned with an integral boundary value problem of fractional differential equations with $p$-Laplacian operator. Sufficient conditions ensuring the existence of extremal solutions for the given problem are obtained. Our results are based on the method of upper and lower solutions and monotone iterative technique.
\end{abstract}

MSC: $34 \mathrm{~B} 15$

Keywords: Monotone iterative; $p$-Laplacian operator; Integral boundary value problem; Upper and lower solutions

\section{Introduction}

This paper studies the existence of extremal solutions for the boundary value problem of a fractional $p$-Laplacian equation with the following form:

$$
\left\{\begin{array}{l}
-D^{\sigma}\left(\phi_{p}\left(-D^{\tau} u(t)\right)\right)=h\left(t, u(t), D^{\tau} u(t)\right), \quad 0<t<1, \\
D^{\tau} u(0)=0, \\
D^{\sigma-1}\left(\phi_{p}\left(-D^{\tau} u(1)\right)\right) \\
\quad=I^{\gamma} k\left(\theta, \phi_{p}\left(-D^{\tau} u(\theta)\right)\right)+d=\frac{1}{\Gamma(\gamma)} \int_{0}^{\theta}(\theta-s)^{\gamma-1} k\left(s, \phi_{p}\left(-D^{\tau} u(s)\right)\right) d s+d, \\
u(0)=0, \quad D^{\tau-1} u(1)=I^{\epsilon} u(\zeta)+e=\frac{1}{\Gamma(\epsilon)} \int_{0}^{\zeta}(\zeta-s)^{\epsilon-1} u(s) d s+e,
\end{array}\right.
$$

where $D^{\tau}$ and $D^{\sigma}$ are the standard Riemann-Liouville fractional derivatives, $I^{\gamma}, I^{\epsilon}$ are the Riemann-Liouville fractional integral, and $1<\sigma, \tau<2, \gamma, \epsilon>1,0<\theta, \zeta<1, d, e \in \mathbb{R}$, $h \in C([0,1] \times \mathbb{R} \times \mathbb{R}, \mathbb{R}), k \in C([0,1] \times \mathbb{R}, \mathbb{R})$. The $p$-Laplacian operator is defined as $\phi_{p}(t)=$ $|t|^{p-2} t, p>1$, and $\left(\phi_{p}\right)^{-1}=\phi_{q}, \frac{1}{p}+\frac{1}{q}=1$.

Recently, much attention has been paid to the study of the existence of extremal solutions, for fractional differential equations with corresponding initial or boundary conditions; see [1-9]. The monotone iterative technique, combined with the method of upper and lower solutions, provides an effective mechanism to prove constructive existence results for nonlinear differential equations, the advantage and importance of the technique needs no special emphasis $[10,11]$. By using the monotone iterative technique, Ahmad

(c) The Author(s) 2019. This article is distributed under the terms of the Creative Commons Attribution 4.0 International License (http://creativecommons.org/licenses/by/4.0/), which permits unrestricted use, distribution, and reproduction in any medium, provided you give appropriate credit to the original author(s) and the source, provide a link to the Creative Commons license, and indicate if changes were made. 
[12] and Alsaedi [13] successfully investigated initial value problems for nonlinear fractional differential equations with fractional derivatives. Han [14] considered the existence of positive solutions for the following problem:

$$
\left\{\begin{array}{l}
D^{\beta}\left(\phi_{p}\left(D^{\alpha} u(t)\right)\right)=\lambda f(u(t)), \quad 0<t<1, \\
u(0)=u^{\prime}(0)=u^{\prime}(1)=0, \quad \phi_{p}\left(D^{\alpha} u(0)\right)=\left(\phi_{p}\left(D^{\alpha} u(1)\right)\right)^{\prime}=0,
\end{array}\right.
$$

where $2<\alpha \leq 3,1<\beta \leq 2$ are real numbers, $\lambda>0$ is a parameter, and $f:(0,+\infty) \rightarrow$ $(0,+\infty)$ is continuous. By using the properties of Green function and the Guo-Krasnosel'skii fixed-point theorem on cones, several existence results of at least one or two positive solutions in terms of different eigenvalue interval are obtained. By means of the monotone iterative method, Wang [15] investigated the fractional integral boundary problem

$$
\left\{\begin{array}{l}
{ }^{C} D^{\alpha} u(t)=f(t, u(t), u(\theta(t)), \quad n<\alpha \leq n+1, n \geq 2, t \in[0,1] \\
u^{\prime}(0)=u^{\prime \prime}(0)=u^{\prime \prime \prime}(0)=\cdots=u^{n}(0)=0 \\
u(0)=\int_{0}^{1} g(s, u(s)) d s+\lambda,
\end{array}\right.
$$

where $\lambda>0$, and $f, g$ are continuous functions. However, the existence results in [10] mainly depend upon a restrictive condition, i.e.,

$$
f(t, u, v) \geq f(t, \bar{u}, \bar{v}) .
$$

It is a critical condition in order to discuss the monotone iterative sequences. Therefore, it is natural to ask whether similar results can be obtained if

$$
f\left(t, u(t), D^{\tau} u(t)\right)-f\left(t, v(t), D^{\tau} v(t)\right) \leq L\left[\phi_{p}\left(-D^{\tau} v(t)\right)-\phi_{p}\left(-D^{\tau} u(t)\right)\right] .
$$

Being directly inspired by Wang [15], the purpose of this paper is to study the nonlinear integral boundary value problem for $p$-Laplacian differential equations. The nonlinear terms $h, k$ are not required to satisfy monotonicity conditions on the unknown function $u$ or their derivatives. The monotone iterative technique combined with the method of upper and lower solutions is applied. In particular, we construct two well-defined monotone iterative sequences of upper and lower solutions and prove that they converge uniformly to the actual solution of the problem.

\section{Preliminaries}

In this section, we deduce some preliminary results which will be used in the next section.

Denote $C_{\tau}[0,1]=\left\{u: u \in C[0,1], D^{\tau} u(t) \in C[0,1]\right\}$ and endow it with the norm $\|u\|_{\tau}=\|u\|+\left\|D^{\tau} u\right\|$, where $\|u\|=\max _{0 \leq t \leq 1}|u(t)|$ and $\left\|D^{\tau} u\right\|=\max _{0 \leq t \leq 1}\left|D^{\tau} u(t)\right|$. Then $\left(C_{\tau}[0,1],\|\cdot\|_{\tau}\right)$ is a Banach space (see $\left.[16]\right)$.

Definition 2.1 A function $u(t) \in C_{\tau}[0,1]$ satisfying $D^{\sigma}\left(\phi_{p}\left(-D^{\tau} u(t)\right)\right) \in C[0,1]$ is called a lower solution of problem (1.1) if

$$
\left\{\begin{array}{l}
-D^{\sigma}\left(\phi_{p}\left(-D^{\tau} u(t)\right)\right) \leq h\left(t, u(t), D^{\tau} u(t)\right), \quad 0<t<1, \\
D^{\tau} u(0)=0, \quad D^{\sigma-1}\left(\phi_{p}\left(-D^{\tau} u(1)\right)\right) \leq I^{\gamma} k\left(\theta, \phi_{p}\left(-D^{\tau} u(\theta)\right)\right)+d, \\
u(0)=0, \quad D^{\tau-1} u(1) \leq I^{\epsilon} u(\zeta)+e .
\end{array}\right.
$$


A function $v(t) \in C_{\tau}[0,1]$ satisfying $D^{\sigma}\left(\phi_{p}\left(-D^{\tau} v(t)\right)\right) \in C[0,1]$ is called an upper solution of problem (1.1) if the above inequalities are reversed.

For the sake of convenience, we now present some assumptions as follows.

$\left(\mathrm{H}_{1}\right)$ Assume that $u_{0}, v_{0} \in C_{\tau}[0,1]$ satisfying $D^{\sigma}\left(\phi_{p}\left(-D^{\tau} u_{0}(t)\right)\right), D^{\sigma}\left(\phi_{p}\left(-D^{\tau} v_{0}(t)\right)\right) \in$ $C[0,1]$ are lower and upper solutions of problem (1.1), respectively, and $u_{0}(t) \leq$ $v_{0}(t), D^{\tau} v_{0}(t) \leq D^{\tau} u_{0}(t), t \in[0,1]$.

$\left(\mathrm{H}_{2}\right)$ There exists a constant $L \in \mathbb{R}$ such that

$$
h\left(t, u(t), D^{\tau} u(t)\right)-h\left(t, v(t), D^{\tau} v(t)\right) \leq L\left[\phi_{p}\left(-D^{\tau} v(t)\right)-\phi_{p}\left(-D^{\tau} u(t)\right)\right]
$$

for $u_{0}(t) \leq u(t) \leq v(t) \leq v_{0}(t), D^{\tau} v_{0}(t) \leq D^{\tau} v(t) \leq D^{\tau} u(t) \leq D^{\tau} u_{0}(t), t \in[0,1]$.

$\left(\mathrm{H}_{3}\right)$ There exists a constant $\mu \geq 0$, such that

$$
k\left(t, \phi_{p}\left(-D^{\tau} v(t)\right)\right)-k\left(t, \phi_{p}\left(-D^{\tau} u(t)\right)\right) \geq \mu\left[\phi_{p}\left(-D^{\tau} v(t)\right)-\phi_{p}\left(-D^{\tau} u(t)\right)\right],
$$

for $u_{0}(t) \leq u(t) \leq v(t) \leq v_{0}(t), D^{\tau} v_{0}(t) \leq D^{\tau} v(t) \leq D^{\tau} u(t) \leq D^{\tau} u_{0}(t), t \in[0,1]$.

$\left(\mathrm{H}_{4}\right) \Gamma(\sigma+\gamma)>\mu \theta^{\sigma+\gamma-1}$.

$\left(\mathrm{H}_{5}\right) 2 \Gamma(\sigma+\gamma)|L|<\Gamma(\sigma)\left[\Gamma(\sigma+\gamma)-\mu \theta^{\sigma+\gamma-1}\right]$.

$\left(\mathrm{H}_{6}\right)$ For any $t \in(0,1)$, we have

$$
\Gamma(2-\sigma) t^{\sigma} L>1-\sigma
$$

and

$$
\Gamma(2-\sigma) \mu \theta^{\gamma}<\Gamma(\gamma)
$$

Lemma 2.1 ([17]) Let $f(t) \in C[0,1], a \in \mathbb{R}$, and $\Gamma(\sigma+\gamma) \neq \mu \theta^{\sigma+\gamma-1}$, then the fractional boundary value problem

$$
\left\{\begin{array}{l}
-D^{\sigma} w(t)=f(t), \quad t \in[0,1], \\
w(0)=0, \\
D^{\sigma-1} w(1)=\mu I^{\gamma} w(\theta)+a=\frac{\mu}{\Gamma(\gamma)} \int_{0}^{\theta}(\theta-s)^{\gamma-1} w(s) d s+a,
\end{array}\right.
$$

is equivalent to

$$
w(t)=\int_{0}^{1} J(t, s) f(s) d s+\frac{a \Gamma(\sigma+\gamma) t^{\sigma-1}}{\Gamma(\sigma)\left[\Gamma(\sigma+\gamma)-\mu \theta^{\sigma+\gamma-1}\right]},
$$

where

$$
J(t, s)=\frac{1}{\Delta} \begin{cases}{\left[\Gamma(\sigma+\gamma)-\mu(\theta-s)^{\sigma+\gamma-1}\right] t^{\sigma-1}} & \\ \quad-\left[\Gamma(\sigma+\gamma)-\mu \theta^{\sigma+\gamma-1}\right](t-s)^{\sigma-1}, & t \leq s \leq \theta ; \\ \Gamma(\sigma+\gamma) t^{\sigma-1}-\mu(\theta-s)^{\sigma+\gamma-1} t^{\sigma-1}, & \theta \leq s \leq t ; \\ \Gamma(\sigma+\gamma)\left[t^{\sigma-1}-(t-s)^{\sigma-1}\right]+\mu \theta^{\sigma+\gamma-1}(t-s)^{\sigma-1}, & s \geq t, s \geq \theta,\end{cases}
$$

and $\Delta=\Gamma(\sigma)\left[\Gamma(\sigma+\gamma)-\mu \theta^{\sigma+\gamma-1}\right]$ 
Lemma 2.2 ([17]) If $\left(\mathrm{H}_{4}\right)$ holds, then the Green's function $J(t, s)$ satisfies

$$
0 \leq J(t, s) \leq \frac{\Gamma(\sigma+\gamma)}{\Gamma(\sigma)\left[\Gamma(\sigma+\gamma)-\mu \theta^{\sigma+\gamma-1}\right]}\left(1+t^{\sigma-1}\right) .
$$

Lemma 2.3 Let $L, a \in \mathbb{R}, w(t), f(t) \in C[0,1]$ and $\left(\mathrm{H}_{4}\right),\left(\mathrm{H}_{5}\right)$ hold, then the boundary value problem

$$
\left\{\begin{array}{l}
-D^{\sigma} w(t)+L w(t)=f(t), \quad t \in[0,1] \\
w(0)=0 \\
D^{\sigma-1} w(1)=\mu I^{\gamma} w(\theta)+a
\end{array}\right.
$$

has a unique solution $w(t) \in C[0,1]$.

Proof It follows from Lemma 2.1 that problem (2.2) is equivalent to the following integral equation:

$$
w(t)=\int_{0}^{1} J(t, s)[f(s)-L w(s)] d s+\frac{a \Gamma(\sigma+\gamma) t^{\sigma-1}}{\Gamma(\sigma)\left[\Gamma(\sigma+\gamma)-\mu \theta^{\sigma+\gamma-1}\right]}, \quad \forall t \in[0,1] .
$$

Let

$$
A w(t)=\int_{0}^{1} J(t, s)[f(s)-L w(s)] d s+\frac{a \Gamma(\sigma+\gamma) t^{\sigma-1}}{\Gamma(\sigma)\left[\Gamma(\sigma+\gamma)-\mu \theta^{\sigma+\gamma-1}\right]}, \quad \forall t \in[0,1] .
$$

For any $u, w \in C[0,1]$, by $\left(\mathrm{H}_{4}\right)$ and Lemma 2.2, we have

$$
\begin{aligned}
\|A u-A w\| & =\max _{0 \leq t \leq 1}|A u(t)-A w(t)| \\
& \leq \max _{0 \leq t \leq 1}\left(\int_{0}^{1} J(t, s)|L| \cdot|u-w| d s\right) \\
& \leq \frac{|L| \Gamma(\sigma+\gamma)\|u-w\|}{\Gamma(\sigma)\left[\Gamma(\sigma+\gamma)-\mu \theta^{\sigma+\gamma-1}\right]} \max _{0 \leq t \leq 1}\left(1+t^{\sigma-1}\right) \\
& \leq \frac{2 \Gamma(\sigma+\gamma)\|u-w\||L|}{\Gamma(\sigma)\left[\Gamma(\sigma+\gamma)-\mu \theta^{\sigma+\gamma-1}\right]} .
\end{aligned}
$$

Noting that $\left(\mathrm{H}_{5}\right)$ holds, which implies $\frac{2 \Gamma(\sigma+\gamma)|L|}{\Gamma(\sigma)\left[\Gamma(\sigma+\gamma)-\mu \theta^{\sigma+\gamma-1}\right]}<1$, we have

$$
\|A u-A w\|<\|u-w\| .
$$

By the Banach fixed point theorem, the operator $A$ has a unique fixed point. That is, (2.2) has a unique solution.

Lemma 2.4 Assume that $z(t) \in C[0,1], k \in \mathbb{R}$. Then the fractional boundary value problem

$$
\left\{\begin{array}{l}
-D^{\tau} u(t)=z(t), \quad 0<t<1, \\
u(0)=0, \quad D^{\tau-1} u(1)=k,
\end{array}\right.
$$


is equivalent to

$$
u(t)=\int_{0}^{1} H(t, s) z(s) d s+\frac{k t^{\tau-1}}{\Gamma(\tau)},
$$

where

$$
H(t, s)= \begin{cases}t^{\tau-1}-(t-s)^{\tau-1}, & 0 \leq s \leq t \leq 1 \\ t^{\tau-1}, & 0 \leq t \leq s \leq 1\end{cases}
$$

Proof We can transform the equation $-D^{\tau} v(t)=z(t)$ to an equivalent integral equation

$$
u(t)=-I^{\tau} z(t)+C_{1} t^{\tau-1}+C_{2} t^{\tau-2} .
$$

Note that $u(0)=0$, we have $C_{2}=0$. Consequently, we have the following form:

$$
u(t)=-I^{\tau} z(t)+C_{1} t^{\tau-1}
$$

and

$$
\begin{aligned}
D^{\tau-1} u(t) & =-D^{\tau-1} I^{\tau} z(t)+C_{1} D^{\tau-1} t^{\tau-1} \\
& =-I^{\tau-(\tau-1)} z(t)+C_{1} D^{\tau-1} t^{\tau-1} \\
& =-\int_{0}^{t} z(s) d s+C_{1} \Gamma(\tau) .
\end{aligned}
$$

On the other hand $D^{\tau-1} u(1)=k$, and we obtain

$$
C_{1}=\frac{1}{\Gamma(\tau)} \int_{0}^{1} z(s) d s+\frac{k}{\Gamma(\tau)} .
$$

Therefore, the solution of problem (2.3) is

$$
\begin{aligned}
u(t) & =-\frac{1}{\Gamma(\tau)} \int_{0}^{t}(t-s)^{\tau-1} z(s) d s+\frac{t^{\tau-1}}{\Gamma(\tau)} \int_{0}^{1} z(s) d s+\frac{k t^{\tau-1}}{\Gamma(\tau)} \\
& =\int_{0}^{1} H(t, s) z(s) d s+\frac{k t^{\tau-1}}{\Gamma(\tau)}
\end{aligned}
$$

Lemma 2.5 Assume that $k, a \in \mathbb{R}, u(t) \in C_{\tau}[0,1], f(t) \in C[0,1]$ and $\left(\mathrm{H}_{4}\right),\left(\mathrm{H}_{5}\right)$ hold. Then the boundary value problem

$$
\left\{\begin{array}{l}
-D^{\sigma}\left(\phi_{p}\left(-D^{\tau} u(t)\right)\right)+L \phi_{p}\left(-D^{\tau} u(t)\right)=f(t), \quad 0<t<1, \\
D^{\tau} u(0)=0, \quad D^{\sigma-1}\left(\phi_{p}\left(-D^{\tau} u(1)\right)\right)=\mu I^{\gamma} \phi_{p}\left(-D^{\tau} u(\theta)\right)+a, \\
u(0)=0, \quad D^{\tau-1} u(1)=k,
\end{array}\right.
$$

has a unique solution $u(t)$. 
Proof Let $\phi_{p}\left(-D^{\tau} u(t)\right)=w(t)$, and consider the boundary value problem:

$$
\left\{\begin{array}{l}
-D^{\sigma} w(t)+L w(t)=f(t), \quad 0<t<1, \\
w(0)=0, \quad D^{\sigma-1} w(1)=\mu I^{\gamma} w(\theta)+a .
\end{array}\right.
$$

From Lemma 2.3, we know that (2.6) has a unique solution $w(t) \in C[0,1]$. Note that $\phi_{p}\left(-D^{\tau} u(t)\right)=w(t) \in C[0,1]$, and $-D^{\tau} u(t)=\phi_{q}(w(t)) \in C[0,1]$. Then the problem (2.5) is transformed into to the following problem:

$$
\left\{\begin{array}{l}
-D^{\tau} u(t)=\phi_{q}(w(t)), \quad 0<t<1 \\
u(0)=0, \quad D^{\tau-1} u(1)=k
\end{array}\right.
$$

By Lemma 2.4, the solution of (2.7) can be written

$$
u(t)=\int_{0}^{1} H(t, s) \phi_{q}(w(s)) d s+\frac{k t^{\tau-1}}{\Gamma(\tau)} .
$$

Combining with (2.6) and (2.7), we assert that the boundary problem (2.5) has a unique solution $u(t)$.

Lemma 2.6 ([18, Lemma 2.6]) Assume that $\left(\mathrm{H}_{6}\right)$ holds, $w(t) \in C[0,1]$, satisfying $D^{\sigma} w(t) \in$ $C[0,1]$ and

$$
\left\{\begin{array}{l}
-D^{\sigma} w(t) \geq-L w(t), \quad t \in[0,1] \\
w(0)=0 \\
D^{\sigma-1} w(1) \geq \mu I^{\gamma} w(\theta),
\end{array}\right.
$$

then $w(t) \geq 0, \forall t \in[0,1]$.

Lemma 2.7 If $u(t) \in C[0,1]$ satisfies

$$
\left\{\begin{array}{l}
-D^{\tau} u(t) \geq 0, \quad 0<t<1 \\
u(0)=0 \\
D^{\tau-1} u(1) \geq 0
\end{array}\right.
$$

then $u(t) \geq 0, \forall t \in[0,1]$.

Proof By Lemma 2.4, we know that (2.3) has a unique solution

$$
u(t)=\int_{0}^{1} H(t, s) z(s) d s+\frac{k t^{\tau-1}}{\Gamma(\tau)} .
$$

It is easy to verify that the Green's function $H(t, s) \geq 0, t, s \in[0,1]$. Let $z(t) \geq 0$ and $k \geq 0$. Then we obtain (2.9) and $u(t) \geq 0, \forall t \in[0,1]$. 


\section{Main results}

Theorem 3.1 Suppose that $\left(\mathrm{H}_{1}\right)-\left(\mathrm{H}_{6}\right)$ hold. Then problem (1.1) has extremal solution $u^{*}, v^{*} \in\left[u_{0}, v_{0}\right]$. Moreover,

$$
u_{0}(t) \leq u^{*}(t) \leq v^{*}(t) \leq v_{0}(t)
$$

and

$$
D^{\tau} v_{0}(t) \leq D^{\tau} v^{*}(t) \leq D^{\tau} u^{*}(t) \leq D^{\tau} u_{0}(t), \quad \forall t \in[0,1]
$$

Proof For $n=0,1,2, \ldots$, we define

$$
\left\{\begin{array}{l}
-D^{\sigma}\left(\phi_{p}\left(-D^{\tau} u_{n+1}(t)\right)\right) \\
\quad=h\left(t, u_{n}(t), D^{\tau} u_{n}(t)\right)-L\left[\phi_{p}\left(-D^{\tau} u_{n+1}(t)\right)-\phi_{p}\left(-D^{\tau} u_{n}(t)\right)\right], \\
D^{\tau} u_{n+1}(0)=0 \\
D^{\sigma-1}\left(\phi_{p}\left(-D^{\tau} u_{n+1}(1)\right)\right) \\
\quad=I^{\gamma}\left\{k\left(\theta, \phi_{p}\left(-D^{\tau} u_{n}(\theta)\right)\right)+\mu\left[\phi_{p}\left(-D^{\tau} u_{n+1}(\theta)\right)-\phi_{p}\left(-D^{\tau} u_{n}(\theta)\right)\right]\right\}+d, \\
u_{n+1}(0)=0, \quad D^{\tau-1} u_{n+1}(1)=I^{\epsilon} u_{n}(\zeta)+e,
\end{array}\right.
$$

and

$$
\left\{\begin{array}{l}
-D^{\sigma}\left(\phi_{p}\left(-D^{\tau} v_{n+1}(t)\right)\right) \\
\quad=h\left(t, v_{n}(t), D^{\tau} v_{n}(t)\right)-L\left[\phi_{p}\left(-D^{\tau} v_{n+1}(t)\right)-\phi_{p}\left(-D^{\tau} v_{n}(t)\right)\right] \\
D^{\tau} v_{n+1}(0)=0 \\
D^{\sigma-1}\left(\phi_{p}\left(-D^{\tau} v_{n+1}(1)\right)\right) \\
\quad=I^{\gamma}\left\{k\left(\theta, \phi_{p}\left(-D^{\tau} v_{n}(\theta)\right)\right)+\mu\left[\phi_{p}\left(-D^{\tau} v_{n+1}(\theta)\right)-\phi_{p}\left(-D^{\tau} v_{n}(\theta)\right)\right]\right\}+d \\
v_{n+1}(0)=0, \quad D^{\tau-1} v_{n+1}(1)=I^{\epsilon} v_{n}(\zeta)+e
\end{array}\right.
$$

In view of Lemma 2.5, the functions $u_{1}$ and $v_{1}$ are well defined. First, we show that $u_{0}(t) \leq u_{1}(t) \leq v_{1}(t) \leq v_{0}(t)$, and $D^{\tau} v_{0}(t) \leq D^{\tau} v_{1}(t) \leq D^{\tau} u_{1}(t) \leq D^{\tau} u_{0}(t), t \in[0,1]$. Let $\delta(t)=\phi_{p}\left(-D^{\tau} u_{1}(t)\right)-\phi_{p}\left(-D^{\tau} u_{0}(t)\right)$. From (3.1) and $\left(\mathrm{H}_{1}\right)$, we obtain

$$
\begin{aligned}
-D^{\sigma} \delta(t) \geq & h\left(t, u_{0}(t), D^{\tau} u_{0}(t)\right)-L\left[\phi_{p}\left(-D^{\tau} u_{1}(t)\right)-\phi_{p}\left(-D^{\tau} u_{0}(t)\right)\right] \\
& -h\left(t, u_{0}(t), D^{\tau} u_{0}(t)\right) \\
\geq & -L \delta(t) .
\end{aligned}
$$

Also $\delta(0)=0$ and

$$
\begin{aligned}
D^{\sigma-1} \delta(1)= & D^{\sigma-1}\left(\phi_{p}\left(-D^{\tau} u_{1}(1)\right)\right)-D^{\sigma-1}\left(\phi_{p}\left(-D^{\tau} u_{0}(1)\right)\right) \\
\geq & I^{\gamma}\left\{k\left(\theta, \phi_{p}\left(-D^{\tau} u_{0}(\theta)\right)\right)+\mu\left[\phi_{p}\left(-D^{\tau} u_{1}(\theta)\right)-\phi_{p}\left(-D^{\tau} u_{0}(\theta)\right)\right]\right\} \\
& -I^{\gamma} k\left(\theta, \phi_{p}\left(-D^{\tau} u_{0}(\theta)\right)\right) \\
\geq & \mu I^{\gamma} \delta(\theta) .
\end{aligned}
$$


In view of Lemma 2.6, we have $\phi_{p}\left(-D^{\tau} u_{1}(t)\right) \geq \phi_{p}\left(-D^{\tau} u_{0}(t)\right), t \in[0,1]$, since $\phi_{p}(x)$ is nondecreasing, thus

$$
D^{\tau} u_{1}(t) \leq D^{\tau} u_{0}(t)
$$

Let $\alpha(t)=u_{1}(t)-u_{0}(t)$, it follows from (3.1) and (3.3) that

$$
\left\{\begin{array}{l}
-D^{\tau} \alpha(t)=-D^{\tau} u_{1}(t)+D^{\tau} u_{0}(t) \geq 0, \quad t \in[0,1] \\
\alpha(0)=0 \\
D^{\tau-1} \alpha(1)=D^{\tau-1} u_{1}(1)-D^{\tau-1} u_{0}(1) \geq I^{\epsilon} u_{0}(\zeta)-I^{\epsilon} u_{0}(\zeta)=0 .
\end{array}\right.
$$

According to Lemma 2.7, we have $u_{1}(t) \geq u_{0}(t), \forall t \in[0,1]$.

By a similar way, we can show that $v_{0}(t) \geq v_{1}(t)$, and $D^{\tau} v_{0}(t) \leq D^{\tau} v_{1}(t)$. Now, we put $p(t)=\phi_{p}\left(-D^{\tau} v_{1}(t)\right)-\phi_{p}\left(-D^{\tau} u_{1}(t)\right)$. From $\left(\mathrm{H}_{2}\right)$ and $\left(\mathrm{H}_{3}\right)$, we have

$$
\begin{aligned}
-D^{\sigma} p(t)= & h\left(t, v_{0}(t), D^{\tau} v_{0}(t)\right)-L\left[\phi_{p}\left(-D^{\tau} v_{1}(t)\right)-\phi_{p}\left(-D^{\tau} v_{0}(t)\right)\right] \\
& -h\left(t, u_{0}(t), D^{\tau} u_{0}(t)\right)+L\left[\phi_{p}\left(-D^{\tau} u_{1}(t)\right)-\phi_{p}\left(-D^{\tau} u_{0}(t)\right)\right] \\
\geq & -L\left[\left(\phi_{p}\left(-D^{\tau} v_{0}(t)\right)-\phi_{p}\left(-D^{\tau} u_{0}(t)\right)\right]-L\left[\phi_{p}\left(-D^{\tau} v_{1}(t)\right)-\phi_{p}\left(-D^{\tau} v_{0}(t)\right)\right]\right. \\
& +L\left[\phi_{p}\left(-D^{\tau} u_{1}(t)\right)-\phi_{p}\left(-D^{\tau} u_{0}(t)\right)\right] \\
= & -L p(t),
\end{aligned}
$$

also $p(0)=0$, and

$$
\begin{aligned}
D^{\sigma-1} p(1)= & I^{\gamma}\left\{k\left(\theta, \phi_{p}\left(-D^{\tau} v_{0}(\theta)\right)\right)+\mu\left[\phi_{p}\left(-D^{\tau} v_{1}(\theta)\right)-\phi_{p}\left(-D^{\tau} v_{0}(\theta)\right)\right]\right\} \\
& -I^{\gamma}\left\{k\left(\theta, \phi_{p}\left(-D^{\tau} u_{0}(\theta)\right)\right)+\mu\left[\phi_{p}\left(-D^{\tau} u_{1}(\theta)\right)-\phi_{p}\left(-D^{\tau} u_{0}(\theta)\right)\right]\right\} \\
\geq & I^{\gamma}\left\{\mu\left[\phi_{p}\left(-D^{\tau} v_{0}(\theta)\right)-\phi_{p}\left(-D^{\tau} u_{0}(\theta)\right)\right]\right. \\
& +\mu\left[\phi_{p}\left(-D^{\tau} v_{1}(\theta)\right)-\phi_{p}\left(-D^{\tau} v_{0}(\theta)\right)\right] \\
& \left.-\mu\left[\phi_{p}\left(-D^{\tau} u_{1}(\theta)\right)-\phi_{p}\left(-D^{\tau} u_{0}(\theta)\right)\right]\right\} \\
= & \mu I^{\gamma} p(\theta) .
\end{aligned}
$$

In view of Lemma 2.6, we have $p(t) \geq 0, \forall t \in[0,1]$. Thus we have $\phi_{p}\left(-D^{\tau} v_{1}(t)\right) \geq$ $\phi_{p}\left(-D^{\tau} u_{1}(t)\right)$, that is, $D^{\tau} v_{1}(t) \leq D^{\tau} u_{1}(t)$, since $\phi_{p}$ is nondecreasing. Therefore $D^{\tau} v_{0}(t) \leq$ $D^{\tau} v_{1}(t) \leq D^{\tau} u_{1}(t) \leq D^{\tau} u_{0}(t) \forall t \in[0,1]$ holds.

Let $\theta(t)=v_{1}(t)-u_{1}(t)$. From $\left(\mathrm{H}_{1}\right)$, we have

$$
\left\{\begin{array}{l}
-D^{\tau} \theta(t)=-D^{\tau} v_{1}(t)+D^{\tau} u_{1}(t) \geq 0, \\
\theta(0)=0, \quad D^{\tau-1} \theta(1)=D^{\tau-1} v_{1}(1)-D^{\tau-1} u_{1}(1)=I^{\epsilon} v_{0}(\zeta)-I^{\epsilon} u_{0}(\zeta) \geq 0 .
\end{array}\right.
$$

Moreover, we get $v_{1}(t) \geq u_{1}(t)$, from Lemma 2.7. Hence, we have the relation $u_{0}(t) \leq$ $u_{1}(t) \leq v_{1}(t) \leq v_{0}(t), \forall t \in[0,1]$. 
In the following, we show that $u_{1}(t), v_{1}(t)$ are lower and upper solutions of problem (1.1), respectively. From (3.1)-(3.2) and $\left(\mathrm{H}_{2}\right),\left(\mathrm{H}_{3}\right)$, one gets

$$
\begin{aligned}
-D^{\sigma}\left(\phi_{p}\left(-D^{\tau} u_{1}(t)\right)\right)= & h\left(t, u_{0}(t), D^{\tau} u_{0}(t)\right)-h\left(t, u_{1}(t), D^{\tau} u_{1}(t)\right)+h\left(t, u_{1}(t), D^{\tau} u_{1}(t)\right) \\
& -L\left[\phi_{p}\left(-D^{\tau} u_{1}(t)\right)-\phi_{p}\left(-D^{\tau} u_{0}(t)\right)\right] \\
\leq & L\left[\phi_{p}\left(-D^{\tau} u_{1}(t)\right)-\phi_{p}\left(-D^{\tau} u_{0}(t)\right)\right] \\
& -L\left[\phi_{p}\left(-D^{\tau} u_{1}(t)\right)-\phi_{p}\left(-D^{\tau} u_{0}(t)\right)\right] \\
& +h\left(t, u_{1}(t), D^{\tau} u_{1}(t)\right) \\
= & h\left(t, u_{1}(t), D^{\tau} u_{1}(t)\right) .
\end{aligned}
$$

Also $D^{\tau} u_{1}(0)=0, u_{1}(0)=0$, and

$$
\begin{aligned}
D^{\sigma-1}\left(\phi_{p}\left(-D^{\tau} u_{1}(1)\right)\right)= & I^{\gamma}\left\{k\left(\theta, \phi_{p}\left(-D^{\tau} u_{0}(\theta)\right)\right)-k\left(\theta, \phi_{p}\left(-D^{\tau} u_{1}(\theta)\right)\right)\right. \\
& +k\left(\theta, \phi_{p}\left(-D^{\tau} u_{1}(\theta)\right)\right) \\
& \left.+\mu\left[\phi_{p}\left(-D^{\tau} u_{1}(\theta)\right)-\phi_{p}\left(-D^{\tau} u_{0}(\theta)\right)\right]\right\}+d \\
\leq & I^{\gamma}\left\{\mu\left[\phi_{p}\left(-D^{\tau} u_{0}(\theta)\right)-\phi_{p}\left(-D^{\tau} u_{1}(\theta)\right)\right]\right. \\
& +\mu\left[\phi_{p}\left(-D^{\tau} u_{1}(\theta)\right)-\phi_{p}\left(-D^{\tau} u_{0}(\theta)\right)\right] \\
& \left.+k\left(\theta, \phi_{p}\left(-D^{\tau} u_{1}(\theta)\right)\right)\right\}+d \\
= & I^{\gamma} k\left(\theta, \phi_{p}\left(-D^{\tau} u_{1}(\theta)\right)\right)+d, \\
D^{\tau-1} u_{1}(1)=I^{\epsilon} u_{0}(\zeta)+ & e \leq I^{\epsilon} u_{1}(\zeta)+e .
\end{aligned}
$$

This proves that $u_{1}(t)$ is a lower solution of the problem (1.1). Similarly, we find that $v_{1}(t)$ is an upper solution of (1.1).

Using mathematical induction, we see that

$$
u_{0}(t) \leq u_{1}(t) \leq \cdots \leq u_{n}(t) \leq \cdots \leq v_{n}(t) \leq \cdots \leq v_{1}(t) \leq v_{0}(t)
$$

and

$$
D^{\tau} v_{0}(t) \leq D^{\tau} v_{1}(t) \leq \cdots \leq D^{\tau} v_{n}(t) \leq \cdots \leq D^{\tau} u_{n}(t) \leq \cdots \leq D^{\tau} u_{1}(t) \leq D^{\tau} u_{0}(t)
$$

for $t \in[0,1]$ and $n=1,2,3, \ldots$.

Since the space of solutions is $C_{\tau}[0,1]$, the sequences $\left\{u_{n}\right\}$ and $\left\{v_{n}\right\}$ are uniformly bounded and equi-continuous. The Arzela-Ascoli theorem guarantees that they are relatively compact sets in the space $C_{\tau}[0,1]$. Therefore, $\left\{u_{n}\right\}$ and $\left\{v_{n}\right\}$ converge, say to $u^{*}(t)$ and $v^{*}(t)$, uniformly on $[0,1]$, respectively. That is,

$$
\lim _{n \rightarrow \infty} u_{n}(t)=u^{*}(t), \quad \lim _{n \rightarrow \infty} v_{n}(t)=v^{*}(t)
$$

and

$$
\lim _{n \rightarrow \infty} D^{\tau} u_{n}(t)=D^{\tau} u^{*}(t), \quad \lim _{n \rightarrow \infty} D^{\tau} v_{n}(t)=D^{\tau} v^{*}(t),
$$


uniformly on $t \in[0,1]$. Moreover, from (3.1) and (3.2), we find that $u^{*}(t)$ and $v^{*}(t)$ are solutions of problem of (1.1).

Finally, we prove that $u^{*}(t), v^{*}(t)$ are the minimal and maximal solutions of problem (1.1), respectively. Let $u(t) \in\left[u_{0}, v_{0}\right]$ be any solution of the problem (1.1). We suppose that $u_{n}(t) \leq u(t) \leq v_{n}(t), D^{\tau} v_{n}(t) \leq D^{\tau} u(t) \leq D^{\tau} u_{n}(t) \forall t \in[0,1]$ for some $n$. Let $y(t)=$ $\phi_{p}\left(-D^{\tau} u(t)\right)-\phi_{p}\left(-D^{\tau} u_{n+1}(t)\right), x(t)=\phi_{p}\left(-D^{\tau} v_{n+1}(t)\right)-\phi_{p}\left(-D^{\tau} u(t)\right)$. Then, by assumptions $\left(\mathrm{H}_{2}\right)$ and $\left(\mathrm{H}_{3}\right)$, we see that

$$
\begin{aligned}
-D^{\sigma} y(t)= & h\left(t, u(t), D^{\tau} u(t)\right)-h\left(t, u_{n}(t), D^{\tau} u_{n}(t)\right) \\
& +L\left[\phi_{p}\left(-D^{\tau} u_{n+1}(t)\right)-\phi_{p}\left(-D^{\tau} u_{n}(t)\right)\right] \\
\geq & -L\left[\phi_{p}\left(-D^{\tau} u(t)\right)-\phi_{p}\left(-D^{\tau} u_{n}(t)\right)\right] \\
& +L\left[\phi_{p}\left(-D^{\tau} u_{n+1}(t)\right)-\phi_{p}\left(-D^{\tau} u_{n}(t)\right)\right] \\
= & -L y(t),
\end{aligned}
$$

also $y(0)=0$, and

$$
\begin{aligned}
D^{\sigma-1} y(1)= & I^{\gamma} k\left(\theta, \phi_{p}\left(-D^{\tau} u(\theta)\right)\right)-I^{\gamma}\left\{k\left(\theta, \phi_{p}\left(-D^{\tau} u_{n}(\theta)\right)\right)\right. \\
& \left.+\mu\left[\phi_{p}\left(-D^{\tau} u_{n+1}(\theta)\right)-\phi_{p}\left(-D^{\tau} u_{n}(\theta)\right)\right]\right\} \\
= & I^{\gamma}\left\{k\left(\theta, \phi_{p}\left(-D^{\tau} u(\theta)\right)\right)-k\left(\theta, \phi_{p}\left(-D^{\tau} u_{n}(\theta)\right)\right)\right. \\
& \left.-\mu\left[\phi_{p}\left(-D^{\tau} u_{n+1}(\theta)\right)-\phi_{p}\left(-D^{\tau} u_{n}(\theta)\right)\right]\right\} \\
\geq & I^{\gamma}\left\{\mu\left[\phi_{p}\left(-D^{\tau} u(\theta)\right)-\phi_{p}\left(-D^{\tau} u_{n}(\theta)\right)\right]\right. \\
& \left.-\mu\left[\phi_{p}\left(-D^{\tau} u_{n+1}(\theta)\right)-\phi_{p}\left(-D^{\tau} u_{n}(\theta)\right)\right]\right\} \\
= & \mu I^{\gamma} y(\theta) .
\end{aligned}
$$

On the other hand

$$
\left\{\begin{array}{l}
-D^{\sigma} x(t) \geq-L x(t) \\
x(0)=0 \\
D^{\sigma-1} x(1) \geq \mu I^{\gamma} x(\theta)
\end{array}\right.
$$

Using Lemma 2.6, we have

$$
D^{\tau} v_{n+1}(t) \leq D^{\tau} u(t) \leq D^{\tau} u_{n+1}(t)
$$

Let $m(t)=u(t)-u_{n+1}(t), \xi(t)=v_{n+1}(t)-u(t)$, by (3.4), we get

$$
\left\{\begin{array}{l}
-D^{\tau} m(t)=-D^{\tau} u(t)+D^{\tau} u_{n+1}(t) \geq 0, \quad t \in[0,1], \\
m(0)=0, \\
D^{\tau-1} m(1)=D^{\tau-1} u(1)-D^{\tau-1} u_{n+1}(1)=I^{\epsilon} u(\zeta)-I^{\epsilon} u_{n}(\zeta) \geq 0,
\end{array}\right.
$$


and

$$
\left\{\begin{array}{l}
-D^{\tau} \xi(t) \geq 0, \quad t \in[0,1] \\
\xi(0)=0 \\
D^{\tau-1} \xi(1) \geq 0
\end{array}\right.
$$

These results and Lemma 2.7 imply that $u_{n+1}(t) \leq u(t) \leq v_{n+1}(t), t \in[0,1]$, so by induction $u^{*}(t) \leq u(t) \leq v^{*}(t), D^{\tau} v^{*}(t) \leq D^{\tau} u(t) \leq D^{\tau} u^{*}(t), t \in[0,1]$ by taking as $n \longrightarrow \infty$. The proof is complete.

\section{Iteration procedure and a numerical example}

In this section, a numerical procedure is introduced to obtain an appropriate solution of (1.1). For a given accuracy $\delta$, we take $u_{n}$ and $v_{n}$ as $\delta$-accurate approximations of $x$ and $y$, respectively, according to the stopping criterion $E(N)<\delta$, where for each $n, E(n)$ is defined by

$$
E(n)=\left\|u_{n}(t)-v_{n}(t)\right\|_{1}=\int_{0}^{1}\left|u_{n}(t)-v_{n}(t)\right| d t .
$$

For the iteration equation (3.1), let $\phi_{p}\left(-D^{\tau} u_{n+1}(t)\right)=x_{n+1}$, and with the boundary conditions $u_{n+1}(0)=0, D^{\tau-1} u_{n+1}(1)=k$, and by Lemma 2.4 ,

$$
u(t)=\frac{k}{\Gamma(\tau)} t^{\tau-1}+\int_{0}^{1} H(t, s) \phi_{q}\left(x_{n+1}(s)\right) d s:=B x_{n+1}(t)
$$

where $k=I^{\epsilon} u_{n}(\zeta)+e=\frac{1}{\Gamma(\epsilon)} \int_{0}^{\zeta}(\zeta-s)^{\epsilon-1} u_{n}(s) d s+e$ and

$$
H(t, s)= \begin{cases}t^{\tau-1}-(t-s)^{\tau-1}, & 0 \leq s \leq t \leq 1 \\ t^{\tau-1}, & 0 \leq t \leq s \leq 1\end{cases}
$$

Thus the iteration equation (3.1) can be rewritten as

$$
\left\{\begin{array}{l}
-D^{\sigma} x_{n+1}=-L x_{n+1}+h\left(t, B x_{n},-\phi_{q} x_{n}\right)+L x_{n}, \\
x_{n+1}(0)=0, \\
\left.\left.D^{\sigma-1} x_{n+1}(1)\right)\right)=\mu I^{\gamma} x_{n+1}(\theta)+a .
\end{array}\right.
$$

Applying Lemma 2.1 to (4.2), we obtain

$$
\begin{aligned}
x_{n+1}(t)= & \frac{a \Gamma(\sigma+\gamma)}{\Gamma(\sigma)\left[\Gamma(\sigma+\gamma)-\mu \theta^{\sigma+\gamma-1}\right]} t^{\sigma-1} \\
& +\int_{0}^{1} J(t, s)\left[-L x_{n+1}(s)+h\left((s), B x_{n}(s),-\phi_{q}\left(x_{n}(s)\right)\right)+L x_{n}(s)\right] d s,
\end{aligned}
$$


where $a=I^{\gamma} k\left(\theta, x_{n}(\theta)\right)-\mu I^{\gamma} x_{n}(\theta)+d$, and

$$
\begin{aligned}
& J(t, s)=\frac{1}{\Delta} \begin{cases}{\left[\Gamma(\sigma+\gamma)-\mu(\theta-s)^{\sigma+\gamma-1}\right] t^{\sigma-1}} & s \leq t, s \leq \theta ; \\
- & {\left[\Gamma(\sigma+\gamma)-\mu \theta^{\sigma+\gamma-1}\right](t-s)^{\sigma-1},} \\
\Gamma(\sigma+\gamma) t^{\sigma-1}-\mu(\theta-s)^{\sigma+\gamma-1} t^{\sigma-1}, & t \leq s \leq \theta ; \\
\Gamma(\sigma+\gamma)\left[t^{\sigma-1}-(t-s)^{\sigma-1}\right]+\mu \theta^{\sigma+\gamma-1}(t-s)^{\sigma-1}, & \theta \leq s \leq t ; \\
\Gamma(\sigma+\gamma) t^{\sigma-1}, & s \geq t, s \geq \theta,\end{cases} \\
& \Delta=\Gamma(\sigma)\left[\Gamma(\sigma+\gamma)-\mu \theta^{\sigma+\gamma-1}\right] .
\end{aligned}
$$

Discretize the interval $[0,1]$ with the nodes $t_{i}=i h, b=\frac{1}{K}, K=\mathbb{N}$. Let $u_{n+1}^{(i)} \approx u_{n+1}\left(t_{i}\right)$, $x_{n+1}^{(i)} \approx x_{n+1}\left(t_{i}\right), H(i, j)=H\left(t_{i}, s_{j}\right), J(i, j)=J\left(t_{i}, s_{j}\right)$ and $h_{n}^{(j)}=h\left(s_{j}, B x_{n}\left(s_{j}\right),-\phi_{q}\left(x_{n}\left(s_{j}\right)\right)\right)+L x_{n}\left(s_{j}\right)$. Using the trapezoidal quadrature rule to approximate the integral in the right hand sides of (4.3) and (4.1), we obtain the following linear systems of equations:

$$
\begin{aligned}
x_{n+1}^{(i)}= & \frac{a \Gamma(\sigma+\gamma)}{\Gamma(\sigma)\left[\Gamma(\sigma+\gamma)-\mu \theta^{\sigma+\gamma-1}\right]} t_{i}^{\sigma-1}-\frac{b}{2} \sum_{j=0}^{K} L J(i, j) d_{j} x_{n+1}^{(j)} \\
& +\frac{b}{2} \sum_{j=0}^{K} J(i, j) d_{j} h_{n}^{(j)}, \quad 0 \leq i \leq K,
\end{aligned}
$$

and

$$
u_{n+1}^{(i)}=B x_{n+1}^{(i)}=\frac{k}{\Gamma(\tau)} t_{i}^{\tau-1}+\frac{b}{2} \sum_{j=0}^{K} H(i, j) d_{j} \phi_{q}\left(x_{n+1}^{(j)}\right), \quad 0 \leq i \leq K,
$$

for the unknown $x_{n+1}^{(i)}, u_{n+1}^{(i)}, 0 \leq i \leq K$, where $\left\{d_{j}\right\}$ are the coefficients in the rule, $d_{0}=d_{K}=$ $1, d_{j}=2$ for $0 \leq i \leq K-1$.

Setting $J_{i j}=\frac{b}{2} J(i, j) d_{j}, H_{i j}=\frac{b}{2} H(i, j) d_{j}$, the matrix $\Phi=\left(J_{i j}\right), A=\mathbb{I}+L \Phi$, and $G=\left(H_{(i j)}\right)$ with $\mathbb{I}$ the identity matrix. The systems (4.4) and (4.5) can be written as a system of matrixvector equations

$$
\left\{\begin{array}{l}
A \vec{x}_{n+1}=\frac{a \Gamma(\sigma+\gamma)}{\Gamma(\sigma)\left[\Gamma(\sigma+\gamma)-\mu \theta^{\sigma+\gamma-1}\right]} S^{\sigma-1}+\vec{F}_{n} \\
\vec{U}_{n+1}=\frac{k}{\Gamma(\tau)} S^{\sigma-1}+G \phi_{q}\left(\vec{x}_{n+1}\right)
\end{array}\right.
$$

where $\vec{x}_{n+1}=\left[x_{n+1}^{(0)}, x_{n+1}^{(1)}, \ldots, x_{n+1}^{(K)}\right], \vec{u}_{n+1}=\left[u_{n+1}^{(0)}, u_{n+1}^{(1)}, \ldots, u_{n+1}^{(K)}\right], S=\left[t_{0}, t_{1}, \ldots, t_{K}\right]^{T}$ and $\vec{F}_{n}$ is a column vector of its component $F_{n}^{i}=\frac{b}{2} \sum_{j=0}^{K} J(i, j) d_{j} h_{n}^{(j)}$.

Example 4.1 Consider the following fractional boundary value problem:

$$
\left\{\begin{array}{l}
-D^{\frac{5}{4}}\left(\phi_{4}\left(-D^{\frac{3}{2}} u(t)\right)\right)=-\frac{1}{2(1+t)^{3}}\left(-D^{\frac{3}{2}} u\right)^{3}+t u, \quad 0<t<1, \\
D^{\frac{3}{2}} u(0)=0, \\
D^{\frac{1}{4}}\left(\phi_{4}\left(-D^{\frac{3}{2}} u(1)\right)\right) \\
\quad=I^{\frac{5}{4}} k\left(\frac{1}{4}, \phi_{4}\left(-D^{\frac{3}{2}} u\left(\frac{1}{4}\right)\right)\right)+0.1 \\
\quad=\frac{1}{\Gamma\left(\frac{5}{4}\right)} \int_{0}^{\frac{1}{4}}\left(\frac{1}{4}-s\right)^{\frac{1}{4}}(s+1)\left(\phi_{4}\left(-D^{\frac{3}{2}} u(s)\right)\right) d s+0.1, \\
u(0)=0, \quad D^{\frac{1}{2}} u(1)=\frac{1}{\Gamma\left(\frac{3}{2}\right)} \int_{0}^{\frac{1}{2}}\left(\frac{1}{2}-s\right)^{\frac{1}{2}} u(s) d s+0.2,
\end{array}\right.
$$


where $\sigma=\frac{5}{4}, \tau=\frac{3}{2}, \gamma=\frac{5}{4}, \epsilon=\frac{3}{2}, \theta=\frac{1}{4}, \zeta=\frac{1}{2}, d=0.1, e=0.2, p=4$, and

$$
\left\{\begin{array}{l}
h\left(t, u, D^{\frac{3}{2}} u\right)=-\frac{1}{2(1+t)^{3}}\left(-D^{\frac{3}{2}} u\right)^{3}+t u, \\
k\left(t, \phi_{4}\left(-D^{\frac{3}{2}} u\right)\right)=(t+1) \phi_{4}\left(-D^{\frac{3}{2}} u\right) .
\end{array}\right.
$$

Take $u_{0}(t)=\frac{1}{2} t^{\frac{1}{2}}, v_{0}(t)=2 t^{\frac{1}{2}}-\frac{\sqrt{\Pi}}{4} t^{2}+\frac{8}{15 \sqrt{\Pi}} t^{\frac{5}{2}}$, then $-1 \leq-t^{\frac{1}{2}}+t=D^{\frac{3}{2}} v_{0}(t) \leq$ $D^{\frac{3}{2}} u_{0}(t)=0$. It is not difficult to verify that $u_{0}, v_{0}$ are lower and upper solutions of problem (4.6), respectively. So $\left(\mathrm{H}_{1}\right)$ holds. In addition, we have

$$
\begin{aligned}
& h\left(t, u(t), D^{\frac{3}{2}} u(t)\right)-h\left(t, v(t), D^{\frac{3}{2}} v(t)\right) \\
& \quad=-\frac{1}{2(1+t)^{3}}\left[-D^{\frac{3}{2}} u\right]^{3}+\frac{1}{2(1+t)^{3}}\left[-D^{\frac{3}{2}} v\right]^{3}+t(u-v) \\
& \quad \leq \frac{1}{2(1+t)^{3}}\left[\left(-D^{\frac{3}{2}} v\right)^{3}-\left(-D^{\frac{3}{2}} u\right)^{3}\right] \\
& \quad \leq \frac{1}{16}\left[\phi_{4}\left(-D^{\frac{3}{2}} v\right)-\phi_{4}\left(-D^{\frac{3}{2}} u\right)\right]
\end{aligned}
$$

and

$$
\begin{aligned}
& k\left(t, \phi_{4}\left(-D^{\frac{3}{2}} v\right)\right)-k\left(t, \phi_{4}\left(-D^{\frac{3}{2}} u\right)\right) \\
& \quad=(t+1)\left[\phi_{4}\left(-D^{\frac{3}{2}} v\right)-\phi_{4}\left(-D^{\frac{3}{2}} u\right)\right] \geq \phi_{4}\left(-D^{\frac{3}{2}} v\right)-\phi_{4}\left(-D^{\frac{3}{2}} u\right),
\end{aligned}
$$

where $u_{0}(t) \leq u(t) \leq v(t) \leq v_{0}(t)$. Therefore $\left(\mathrm{H}_{2}\right)$ and $\left(\mathrm{H}_{3}\right)$ hold.

From (4.7) and (4.8), we have $L=\frac{1}{16}, \mu=1$. Then

$$
\begin{aligned}
& \Gamma(\sigma+\gamma)=\Gamma\left(\frac{5}{4}+\frac{5}{4}\right)=\Gamma\left(\frac{5}{2}\right) \approx 1.3293>\mu \theta^{\sigma+\gamma-1}=1 \cdot\left(\frac{1}{4}\right)^{\frac{3}{2}}=0.125 \\
& \begin{aligned}
2 \Gamma(\sigma+\gamma)|L| & =2 \cdot \Gamma\left(\frac{5}{2}\right) \cdot \frac{1}{16} \approx 0.1662<\Gamma(\sigma)\left[\Gamma(\sigma+\gamma)-\mu \theta^{\sigma+\gamma-1}\right] \\
& =\Gamma\left(\frac{5}{4}\right)\left[\Gamma\left(\frac{5}{2}\right)-1 \cdot\left(\frac{1}{4}\right)^{\frac{3}{2}}\right] \approx 1.1609
\end{aligned} \\
& \Gamma(2-\sigma) \cdot t^{\sigma} \cdot L=\Gamma\left(\frac{3}{4}\right) \cdot t^{\sigma} \cdot \frac{1}{16}>1-\sigma=-\frac{1}{4} \\
& \Gamma(2-\sigma) \mu \theta^{\gamma}=\Gamma\left(\frac{3}{4}\right) \cdot 1 \cdot\left(\frac{1}{4}\right)^{\frac{5}{4}} \approx 0.2332<\Gamma(\gamma)=\Gamma\left(\frac{5}{4}\right) \approx 0.9064
\end{aligned}
$$

It appears that $\left(\mathrm{H}_{4}\right),\left(\mathrm{H}_{5}\right)$ and $\left(\mathrm{H}_{6}\right)$ hold. By Theorem 3.1, the boundary value problem (4.6) has extremal solutions in $\left[u_{0}(t), v_{0}(t)\right]$.

Applying the numerical scheme to Example 4.1, we obtain approximate solutions to $u_{n}$ and $v_{n}$ for $1 \leq n \leq N$, with $N$ some integer. The graphs of to $u_{n}$ and $v_{n}$ for $n=0,1,2,3,4,34$ are shown in Fig. 1. Computed error values $E(n)$ are displayed in Table 1. We found that, for $\delta=10^{-10}$, it took $N=34$ iterations for $E(N)<\delta$. 

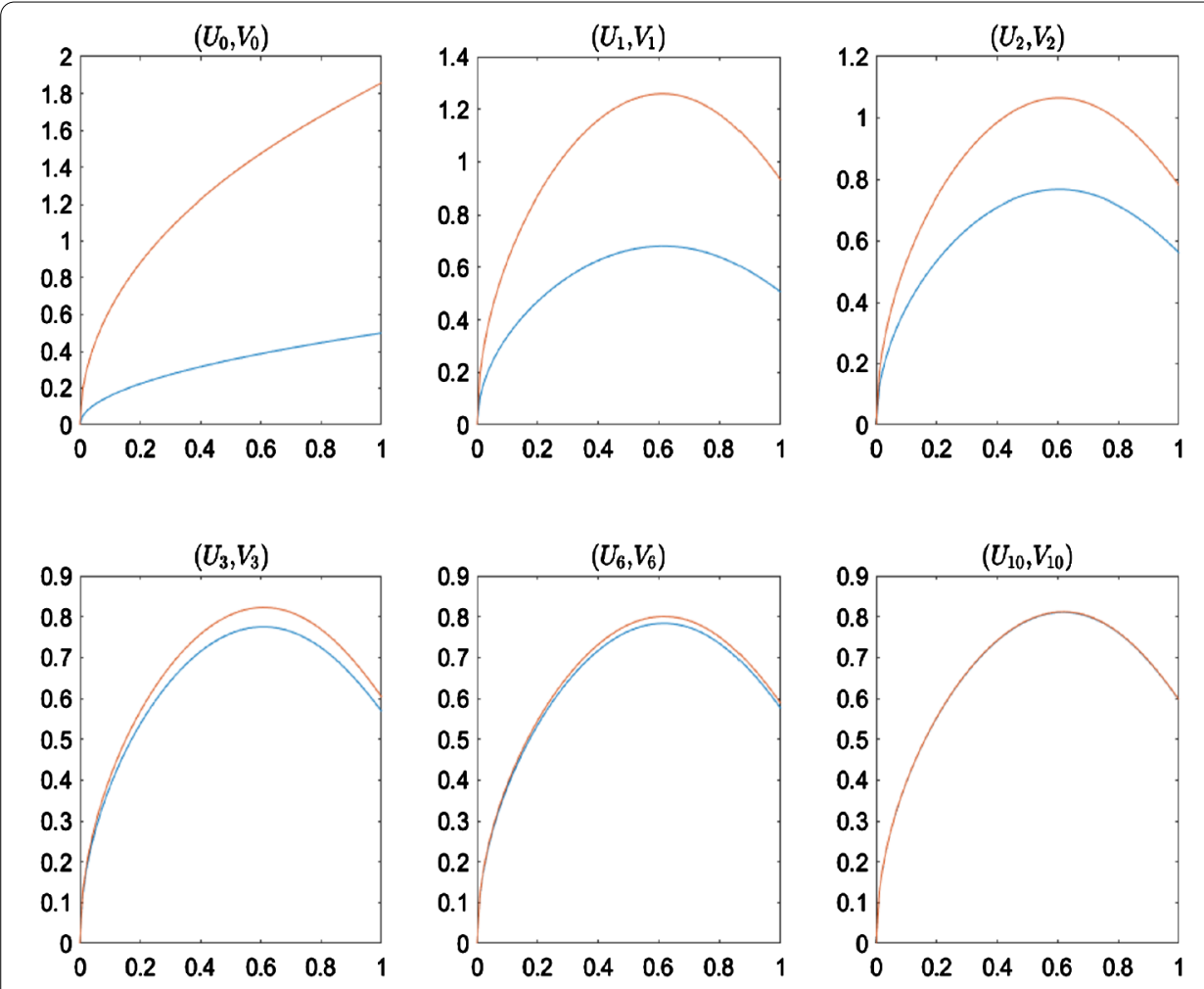

Figure 1 Graph $U_{n}$ and $V_{n}$

Table $1 E(n)=6 n+4, n=0,1,2,3,4,5$

\begin{tabular}{lllllll}
\hline$n$ & 4 & 10 & 16 & 22 & 28 & 34 \\
$E(n)$ & 0.2448 & $3.2744 \mathrm{e}-05$ & $2.3971 \mathrm{e}-07$ & $1.9264 \mathrm{e}-09$ & $1.5492 \mathrm{e}-10$ & $1.2416 \mathrm{e}-11$ \\
\hline
\end{tabular}

\section{Acknowledgements}

The authors sincerely thank the editor and reviewers for their valuable suggestions and useful comments to improve the manuscript.

Funding

This work is supported by Fostering Foundation of Northeast Petroleum University (No. 2017PYYL-08).

\section{Competing interests}

The authors declare to have no competing interests.

\section{Authors' contributions}

The authors have equally made contributions. All authors read and approved the final manuscript.

\section{Publisher's Note}

Springer Nature remains neutral with regard to jurisdictional claims in published maps and institutional affiliations.

Received: 16 November 2018 Accepted: 27 August 2019 Published online: 30 September 2019

\section{References}

1. Wang, J., Xiang, H.: Upper and lower solutions method for a class of singular fractional boundary value problems with p-Laplacian operator. Abstr. Appl. Anal. 2010, Article ID 971824 (2010)

2. Lu, H., Han, Z., Sun, S.: Multiplicity of positive solutions for Sturm-Liouville boundary value problems of fractional differential equations with $p$-Laplacian. Bound. Value Probl. 2014, 26 (2014)

3. Cabada, A., Hamdi, Z.: Nonlinear fractional differential equations with integral boundary value conditions. Appl. Math Comput. 228, 251-257 (2014)

4. Zhang, X., Wang, L., Sun, Q.: Existence of positive solutions for a class of nonlinear fractional differential equations with integral boundary conditions and a parameter. Appl. Math. Comput. 226, 708-718 (2014) 
5. Zhang, $X ., L i u, L ., W u, Y .:$ The uniqueness of positive solution for a fractional order model of turbulent flow in a porous medium. Appl. Math. Lett. 37, 26-33 (2014)

6. Zhang, X., Liu, L., Wiwatannapataphee, B., Wu, Y.: The eigenvalue for a class of singular $p$-Laplacian fractional different equations involving the Riemann-Stieltjes integral boundary condition. Appl. Math. Comput. 235, 412-422 (2014)

7. He, Y.: Extremal solutions for a coupled system of nonlinear fractional differential equations with $p$-Laplacian operator. J. Comput. Anal. Appl. 27, 671-680 (2019)

8. Wang, Y., Liu, L., Zhang, X., Wu, Y.: Positive solutions for an abstract fractional semipositone differential system model for bioprocesses of HIV infection. Appl. Math. Comput. 258, 312-324 (2015)

9. Ahmad, B., Nieto, J.J.: Existence results for nonlinear boundary value problems of fractional integrodifferential equations with integral boundary conditions. Bound. Value Probl. 2009, Article ID 708576 (2009)

10. Ladde, G.S., Lakshmikantham, V., Vatsala, A.S.: Monotone Iterative Techniques for Nonlinear Differential Equations. Pitman, Boston (1985)

11. Nieto, J.J: An abstract monotone iterative technique. Nonlinear Anal., Theory Methods Appl. 28, 1923-1933 (1997)

12. Ahmad, B., Alghanmi, M., Nieto, J.., Alsaedi, A.: On impulsive nonlocal integro-initial value problems involving multi-order Caputo-type generalized fractional derivatives and generalized fractional integrals. Adv. Differ. Equ. 2019 247 (2019)

13. Alsaedi, A., Ahmad, B., Alghanmi, M.: Extremal solutions for generalized Caputo fractional differential equations with Stieltjes-type fractional integro-initial conditions. Appl. Math. Lett. 91, 113-120 (2019)

14. Han, Z., Lu, H., Zhang, C.: Positive solutions for eigenvalue problems for fractional differential equation with generalized p-Laplacian. Appl. Math. Comput. 257, 526-536 (2015)

15. Zhang, L., Ahmad, B., Wang, G.: Explicit iterations and extremal solutions for fractional differential equations with nonlinear integral boundary conditions. Appl. Math. Comput. 268, 388-392 (2015)

16. Tian, Y., Nieto, J.J.: The applications of critical-point theory to discontinuous fractional-order differential equations. Proc. Edinb. Math. Soc. 60, 1021-1051 (2017)

17. Wang, G.: Explicit iteration and unbounded solutions for fractional integral boundary value problem on an infinite interval. Appl. Math. Lett. 17, 1-7 (2015)

18. He, Y:: Existence results and the monotone iterative technique for nonlinear fractional differential systems involving fractional integral boundary conditions. Adv. Differ. Equ. 2017, 264 (2017)

\section{Submit your manuscript to a SpringerOpen ${ }^{0}$ journal and benefit from:}

- Convenient online submission

- Rigorous peer review

- Open access: articles freely available online

- High visibility within the field

- Retaining the copyright to your article

Submit your next manuscript at $\boldsymbol{~ s p r i n g e r o p e n . c o m ~}$ 